\title{
Jejunal Lipoma Concealing Intestinal Adenocarcinoma: Don't Always Regard CT Imaging Features Like Lipoma as Benign or Pseudomalignant Features
}

\author{
Zhang Shuo and LU Bin
}

\begin{abstract}
Lipomas are benign submucosal tumors composed of mature adipose tissue. Some authors have described patients with persistent abdominal pain who were misdiagnosed as intestinal adenocarcinoma and the diagnosis turned out to be a lipoma on histological examination. We discuss the case of a 57-year-old woman presenting with abdominal pain for 4 weeks. Intestinal adenocarcinoma was found by double-balloon enteroscopy (DBE), but at first only lipoma was found by CT scan. Therefore we want to emphasize that lipoma should not always be regarded as pseudomalignant features, sometimes lipoma conceals intestinal adenocarcinoma, and DBE may be a better way to discover malignancy.
\end{abstract}

Key words: lipoma, adenocarcinoma, submucosal tumors, intestinal obstruction, intussusception

(Intern Med 51: 181-184, 2012)

(DOI: 10.2169/internalmedicine.51.6497)

\section{Introduction}

Lipomas are benign submucosal tumors composed of mature adipose tissue. They occur anywhere in the gastrointestinal tract, but the incidence of lipomas occurring in both the small intestine and colon is rare (1-3). Although most intestinal lipomas are usually asymptomatic and detected incidentally, they are even a rarer source of severe symptoms such as obstruction, intussusception, and gastrointestinal bleeding. Those larger than $2 \mathrm{~cm}$ may occasionally cause abdominal pain, changes of bowel habits, rectal bleeding and bowel obstruction, intussusception or prolapse (4-6). Some reports have described patients with persistent abdominal pain who underwent laparoscopic right hemicolectomy for the presumptive endoscopic diagnosis of intestinal adenocarcinoma that turned out to be a lipoma on histological examination $(7,8)$. However, lipomas causing small intestinal intussusception could conceal intestinal adenocarcinoma. Misdiagnoses were inevitable in some cases by regarding the lipoma as benign or by pseudomalignant features.

We describe a patient with persistent abdominal pain who underwent partial enterectomy for the presumptive endoscopic diagnosis of multiple lipomas that turned out to be an adenocarcinoma on histological examination.

\section{Case Report}

A 57-year-old woman presented with abdominal pain and vomiting for a duration of 4 weeks. She denied melena, fever, or chills and her medical history did not include the consumption of alcohol or non-steroid anti-inflammatory drugs (NSAIDs). She had had symptoms intermittently for about 4 weeks. Then, the patient went to local hospital to test CT scan of abdomen. It showed the juncture of duodenum and jejunum with mural thickening and stratification, resulting in the target sign; furthermore, many round masses of fat density representing lipomas were found as the leading point of incomplete intestinal obstruction or intussusception in the jejunum and ileum. The doctor diagnosed the patient as having intestinal intussusception as a result of small intestinal lipomas and gave her traditional Chinese medicine (TCM) for treatment. There was no abdominal pain and vomiting after TCM treatment for a week. However, vomiting and abdominal pain appeared again 3 weeks previously. 

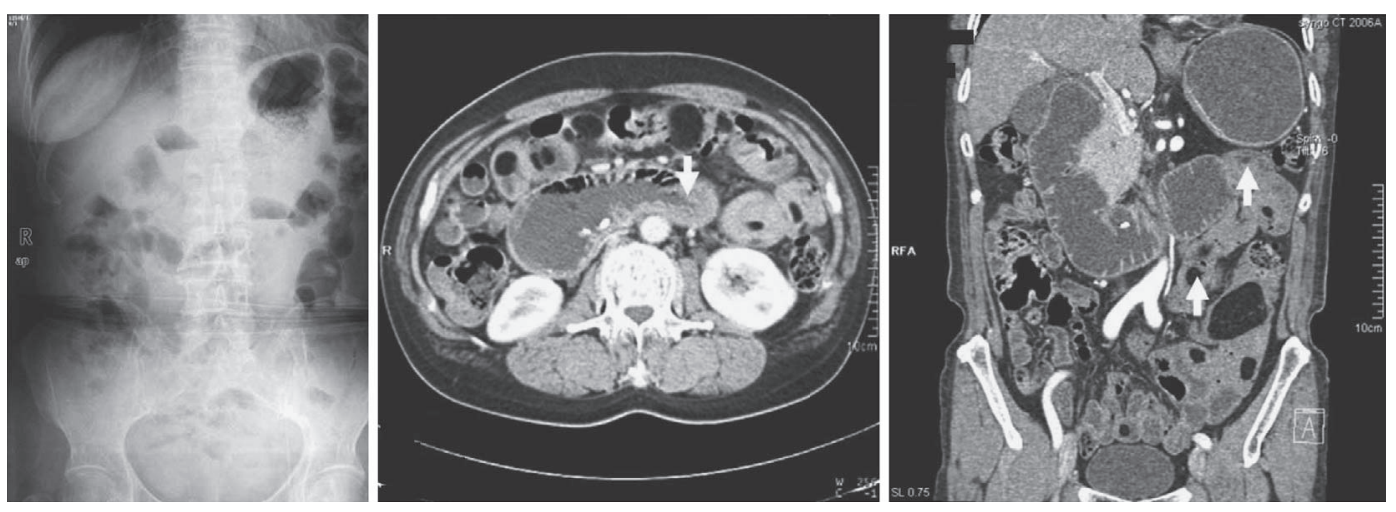

Figure 1. The abdominal X-ray showed incomplete intestinal obstruction with pneumatosis and air-liquid in part of the small intestine. CT scan showed obstruction in the jejunum, resulting in the target sign; lipomas were found as the leading point of incomplete intestinal obstruction or intussusception within the lumen of the intussusceptum in the jejunum, ileum and ascending colon.

Over the recent 2 weeks, the episodes of pain became more pronounced and the patient was not using any specific medication. So the patient came to our hospital.

After admission, physical examination revealed a temperature of $37^{\circ}$, a pulse rate of 80 beats per minute (bpm), a blood pressure of $120 / 80 \mathrm{mmHg}$, and a respiration rate of 18 breaths per minute. The patient was pale-looking with abdominal tenderness in the right lower abdominal quadrant, without guarding or rebound and norm active bowel sounds were auscultated. Examination of the heart and lungs revealed no abnormal findings. Rectal examination revealed tarry fecal content. Turbid gastric content with chyme and bile were obtained on nasogastric aspiration. The initial laboratory workup was as follows: hemoglobin, $12.8 \mathrm{~g} / \mathrm{dL}$; white blood cells, $4,900 / \mathrm{mm}^{3}$ with a normal differential count; platelets, $200,000 / \mathrm{mm}^{3}$; blood glucose, $5.7 \mathrm{mmol} / \mathrm{L}$; blood urea, $5.0 \mathrm{mmol} / \mathrm{L}$; creatinine, $76 \mu \mathrm{mol} / \mathrm{L}$; SGOT, 22 IU/L; SGPT, 28 IU/L; LDH, 135 IU/L; total bilirubin, 11.7 $\mu \mathrm{mol} / \mathrm{L}$; direct bilirubin, $2.7 \mu \mathrm{mol} / \mathrm{L}$; and $\mathrm{Na}^{+}, 136 \mathrm{mmol} / \mathrm{L}$; $\mathrm{K}^{+}$, $3.9 \mathrm{mmol} / \mathrm{L}$; CEA $3.2 \mathrm{ng} / \mathrm{mL}$, CA199 $65.5 \mathrm{U} / \mathrm{mL}$, higher than normal. Fecal occult blood test was negative. A gastrointestinal cause was sought in order to explain the patient's abdominal pain and vomiting. She underwent gastroscopic examination without identification of a specific vomiting etiology; abdominal plain film radiography revealed incomplete intestinal obstruction with pneumatosis and airliquid in part of the small intestine. A CT scan (plain, enhanced) of the abdomen and pelvis revealed obstruction in the juncture of duodenum and jejunum with mural thickening and stratification, resulting in the target sign; furthermore, many round masses of fat density representing lipoma were found as the leading point of incomplete intestinal obstruction or intussusception within the lumen of the intussusceptum in the jejunum, ileum and ascending colon (Fig. 1). It was assumed to be the underlying cause of intestinal obstruction in the juncture of the duodenum and jejunum with intussusception at the lower part of juncture. Multiple lipomas were assumed to be the underlying cause of recurrent partial intestinal obstruction and intussusception in the jejunum, ileum and ascending colon. By double-balloon enterscopy (DBE) reaching somewhere in the jejunum through the mouth, a hard and constrictive lesion was found (Fig. 2) which nearly obstructed the whole lumen and interrupted endoscopy. Biopsies were carried out and the histopathological examination suggested severe dysplasia (Dys III) of intestinal mucosa.

At surgery, a $2.5 \mathrm{~cm} \times 1.5 \mathrm{~cm} \times 1.5 \mathrm{~cm}$ hard and constrictive lesion, which had caused the patient's vomiting, associated with numerous areas of ulceration on its surface and invading the serosa was seen to arise from the intestine at 5 $\mathrm{cm}$ proximal from Treitz ligament and about 20 lipomatous solid mass beads (diameter of $2-3 \mathrm{~cm}$ ) were found nearby (Fig. 3A). The lesion nearly obstructed the whole lumen, and resulted in the dilatation of the proximal small intestine. Pathologic evaluation of the specimen confirmed the diagnosis of an ulcer-infiltrating moderately differentiated adenocarcinoma (Fig. 4A). Segmental resection of the jejunal loop harboring the lesion was carried out and an end-to-end jejunum-jejunum anastomosis was performed. Intraoperative endoscopy (IOE) was performed at the time of the excision of the round masses in the jejunum, ileum and ascending colon (Fig. 3B). Pathologic evaluation of the specimen confirmed the diagnosis of multiple lipomas measuring 1.0-3.0 $\mathrm{cm}$ in diameter and revealed neither heterotrophic gastric and pancreatic tissue nor signs of malignancy (Fig. 4B). Ten days after intervention, the patient was discharged without any complication.

\section{Discussion}

Lipoma of the gastrointestinal tract was first described by Bauer in 1757 (9). Lipomas are rare benign tumors of the small intestine with no malignant potential which are mostly encountered incidentally during investigation of the gastrointestinal tract for another reason, since they are usually asymptomatic (10). As small intestinal lipoma is relatively infrequent, it is even a rarer source of vomiting and gastrointestinal bleeding. Although the majority of these lesions are 

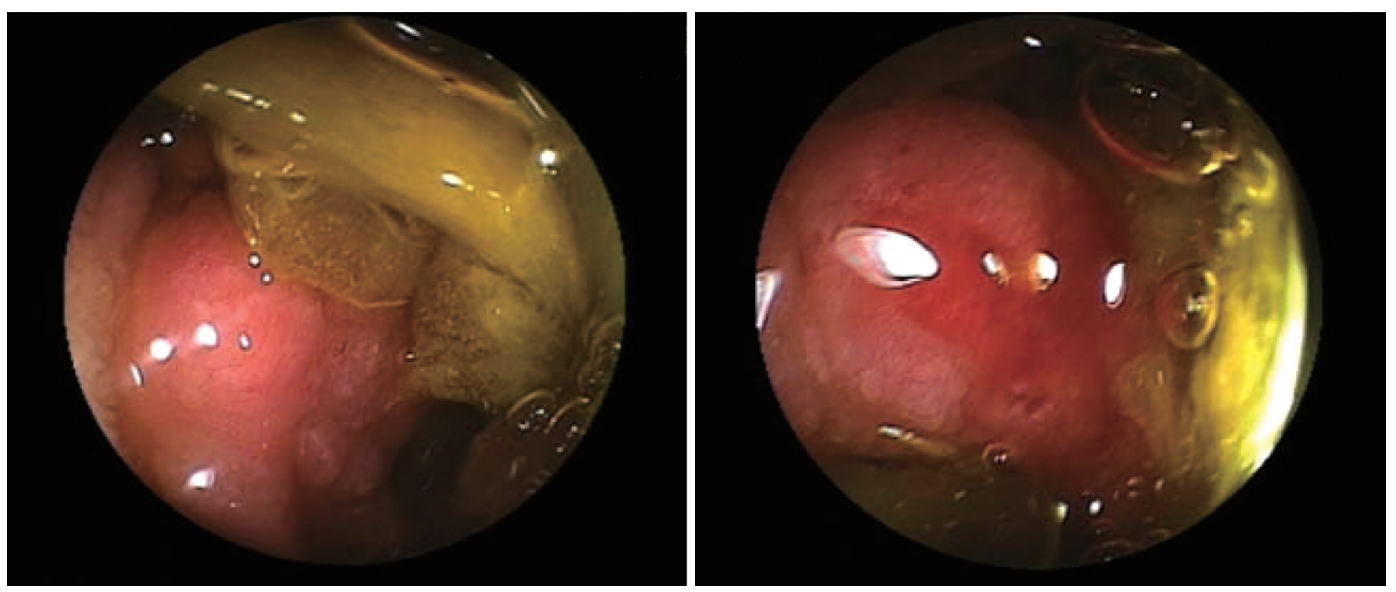

Figure 2. DBE reached somewhere in the jejunum; by an oral route, a hard and constrictive lesion can been seen.
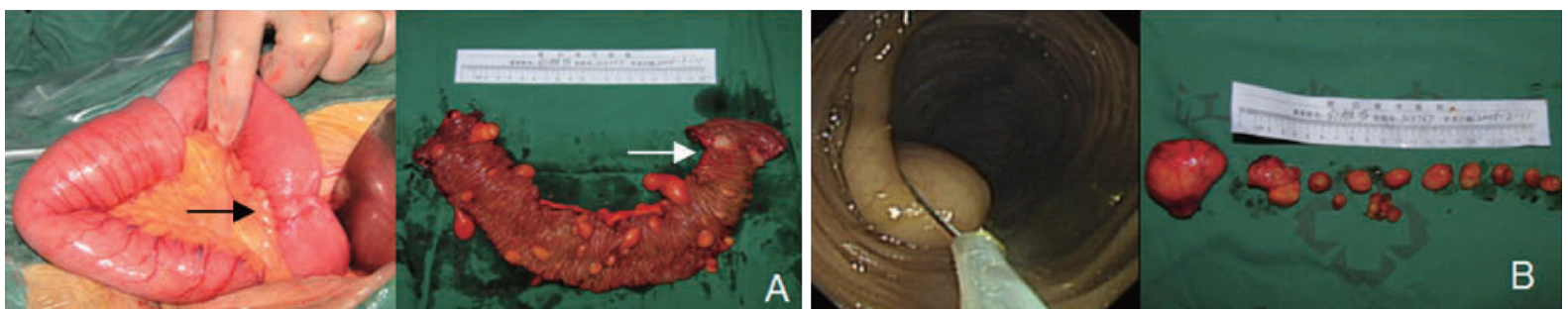

Figure 3. A: $2.5 \mathrm{~cm} \times 1.5 \mathrm{~cm} \times 1.5 \mathrm{~cm}$ hard and constrictive lesion with numerous areas of ulceration on its surface can be seen as well as invasion of the serosa with about 20 lipomatous solid mass beads (diameter of $2-3 \mathrm{~cm}$ ) nearby. B: Intraoperative endoscopy (IOE) was performed at the time of excision of the round masses in the jejunum, ileum and ascending colon.

asymptomatic and detected incidentally during routine examinations or in a surgical specimen removed for various other reasons, on rare occasions they might present with symptoms depending on their size and location. The pathophysiology of the disease process leads to a complicated and confusing clinical picture of recurrent obstructive symptoms, chronic abdominal pain and lower gastrointestinal bleeding that may cause a delay in diagnosis and waste time. Lipomas of the small intestine are the second most common benign tumors next to leiomyomas (11). Their location and the peak age vary, however approximately $50 \%$ are found in the ileum, and the sixth to seventh decades of life are considered to be the most risky period $(10,11)$. In general, lipomas are defined to originate in the submucosal layer and are usually solitary, with variable sizes ranging from $1 \mathrm{~cm}$ to 30 $\mathrm{cm}$ (12). They usually appear as a sessile protrusion into the lumen of the intestine. Multiple lipomas are rare and noted only in $10-20 \%$ of cases. They can cause symptoms when the size exceeds $2 \mathrm{~cm}$ including bleeding with anemia, constipation, change in bowel habits, abdominal pain, intestinal obstruction and rarely intussusception. Lipomas of the large bowel are uncommon fatty neoplasms with a reported incidence ranging between $0.2 \%$ and $4.4 \%$ (13).

Adenocarcinoma of the small intestine is a relatively rare malignancy as compared to the other malignancies of the gastrointestinal tract, but it represents $50 \%$ of small intesti- nal malignant tumors. The clinical signs and symptoms may vary with the tumor site, size, and existence of ulceration. The common presenting signs and symptoms in the present patient were nausea, vomiting, abdominal pain, melena, weight loss, anemia, and a palpable mass, none of which was pathognomonic for small bowel tumors. Nonspecific presentation and infrequent occurrence often lead to a delay in diagnosis and consequent poor prognosis. In recent years, the diagnosis for small intestinal malignancy has been improved as a result of advances in endoscopic technologies such as DBE (14). DBE is a new technique that enables deep intubation of the endoscope into the small intestine; it is a safe procedure and has a high diagnostic and therapeutic yield to make a preoperative histological diagnosis of intestinal malignancy. Confirming malignancy using DBE in equivocal cases may greatly increase both the compliance of patients for an operation and confidence of the physician planning a surgical resection.

Some authors have described patients with persistent abdominal pain who underwent laparoscopic right hemicolectomy for the presumptive endoscopic diagnosis of intestinal adenocarcinoma that turned out to be a lipoma upon histological examination $(7,8)$. But, in the present case, the doctor of the local hospital thought the obstructive periods and abdominal pain of the patient might be attributed to luminal obstruction which might be induced by lipomatous develop- 


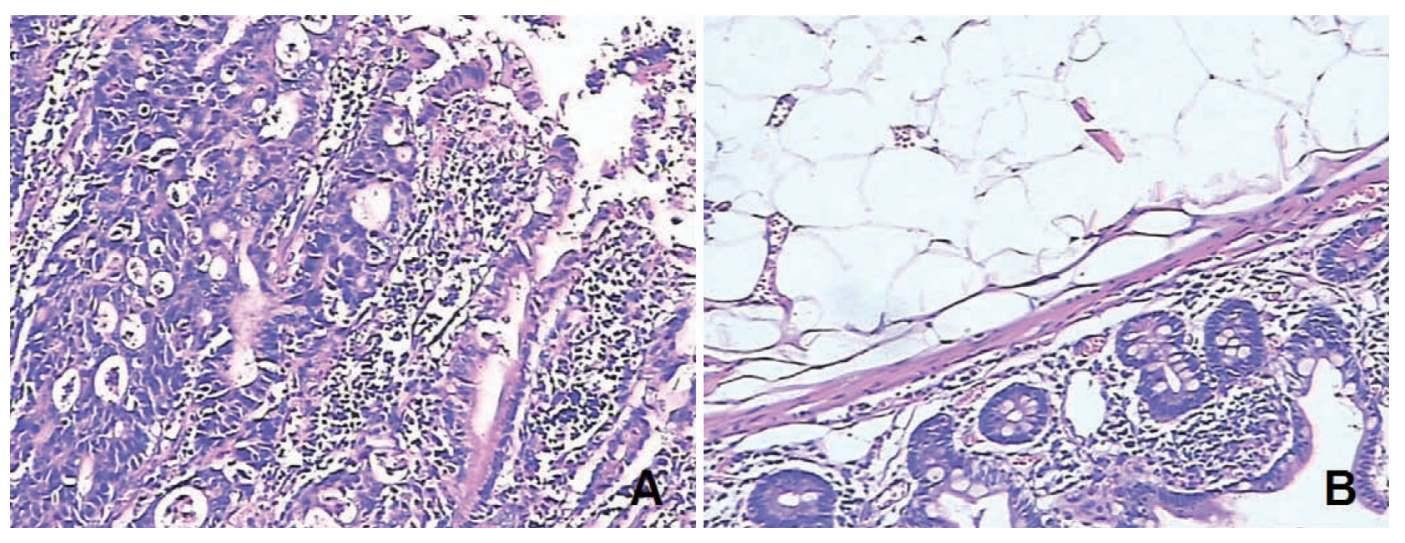

Figure 4. A: moderately differentiated adenocarcinoma: Oncocyte was noted to be tubiform and it invaded the serosa. Cancer embolus could be seen in the vasculature. Surgical margin was negative. Lymph node metastasis: 2/18 mesenteric lymph nodes were positive. B: small intestinal lipomas.

ment. As lipomas are non-malignant diseases, the doctor might not have considered that it may combine with other malignant diseases and did not submit the patient to further examinations such as DBE, just gave her conservative medical management. According to the CT scan, the lipomas of the patient were not big enough to fill to the full coelenterons. So, if the intussusception was only induced by lipomas, the symptoms could be relieved by conservative medical management. As the abdominal pain was aggravated after TCM treatment, we considered this symptomatology might be the probable intussusception based on not only lipomas but also for other reasons. That is why we repeated the CT scan and did DBE. Because the lipomas surrounded the jejunal adenocarcinoma, we could not discover the malignancy through CT scan. Therefore, DBE can sometimes be more useful than CT scan to reveal concealed malignant diseases. Based on the experience with the present case, we consider that it is important to emphasize that when encountering a patient with intestinal lipomas, we should not immediately consider it as non-malignant. We should consider together all of the symptoms and the history. If the patient has other suspicious diseases, DBE might be a good method to clarify a diagnosis.

\section{Conclusion}

Some reports have described patients who underwent laparoscopic right hemicolectomy for the presumptive endoscopic diagnosis of intestinal adenocarcinoma that turned out to be a lipoma on histological examination. However, sometimes lipomas can co-exist with intestinal malignancy. CT scan is sometimes not sufficient to discover it, for this, it may be recommended to consider DBE which might be a better way to find the malignancy.

The authors state that they have no Conflict of Interest (COI).

\section{References}

1. Kim CY, Bandres D, Tio TL, Benjamin SB, Al-Kawas FH. Endoscopic removal of large colonic lipomas. Gastrointest Endosc 55: 929-931, 2002.

2. Wiech T, Walch A, Werner M. Histopathological classification of nonneoplastic and neoplastic gastrointestinal submucosal lesions. Endoscopy 37: 630-634, 2005.

3. Yu HG, Ding YM, Tan S, Luo HS, Yu JP. A safe and efficient strategy for endoscopic resection of large, gastrointestinal lipoma. Surg Endosc 21: 265-269, 2007.

4. Vecchio R, Ferrara M, Mosca F, Ignoto A, Latteri F. Lipomas of the large bowel. Eur J Surg 162: 915-919, 1996.

5. Pfeil SA, Weaver MG, Abdul-Karim FW, Yang P. Colonic lipomas: outcome of endoscopic removal. Gastrointest Endosc 36: 435-438, 1990.

6. Rogy MA, Mirza D, Berlakovich G, Winkelbauer F, Rauhs R. Submucous large-bowel lipomas--presentat ion and management. An 18-year study. Eur J Surg 157: 51-55, 1991.

7. Martin P, Sklow B, Adler DG. Large colonic lipoma mimicking colon cancer and causing colonic intussusception. Dig Dis Sci 53: 2826-2827, 2008.

8. Katsinelos P, Chatzimavroudis G, Zavos C, et al. Cecal lipoma with pseudomalignant features: a case report and review of the literature. World J Gastroenterol 13: 2510-2513, 2007.

9. Ryan J, Martin JE, Pollock DJ. Fatty tumours of the large intestine: a clinicopathological review of 13 cases. Br J Surg 76: 793796, 1989.

10. Fernandez MJ, Davis RP, Nora PF. Gastrointestinal lipomas. Arch Surg 118: 1081-1083, 1983.

11. Olmsted WW, Ros PR, Hjermstad BM, et al. Tumors of the small intestine with little or no malignant predisposition: a review of the literature and report of 56 cases. Gastrointest Radiol 12: 231-239, 1987.

12. Zografos G, Tsekouras DK, Lagoudianakis EE, et al. Small intestinal lipoma as a cause of massive gastrointestinal bleeding identified by intraoperative enteroscopy. A case report and review of the literature. Dig Dis Sci 50: 2251-2254, 2005.

13. Vecchio R, Ferrara M, Mosca F, et al. Lipomas of the large bowel. Eur J Surg 162: 915-919, 1996.

14. Yamagami H, Oshitani N, Hosomi S, et al. Usefulness of doubleballoon endoscopy in the diagnosis of malignant small-bowel tumors. Clin Gastroenterol Hepatol 6: 1202-1205, 2008.

(C) 2012 The Japanese Society of Internal Medicine http://www.naika.or.jp/imindex.html 